\title{
Oxaliplatin, irinotecan, and fluorouracil/folinic acid in advanced gastric cancer: a multicenter phase II trial of the Southern Italy Cooperative Oncology Group
}

\author{
Pasquale Comella $\cdot$ Vito Lorusso $\cdot$ Luigi Maiorino $\cdot$ Rossana Casaretti $\cdot$ Michele Cannone $\cdot$ \\ Bruno Massidda $\cdot$ Carlo Putzu $\cdot$ Silvana Leo $\cdot$ Mario Roselli $\cdot$ Sergio Mancarella $\cdot$ \\ Sergio Palmeri · Ettore Greco · Giacomo Vessia · Claudia Sandomenico · Luca Franco
}

Received: 28 November 2008 / Accepted: 9 January 2009 / Published online: 3 February 2009

(C) Springer-Verlag 2009

\begin{abstract}
Purpose This phase II trial assessed the tolerability and efficacy of a triplet of oxaliplatin, irinotecan, and fluorouracil/folinic acid in advanced gastric cancer.

Methods Patients with unresectable or metastatic gastric cancer, unexposed to palliative chemotherapy, received oxaliplatin $85 \mathrm{mg} / \mathrm{m}^{2}$ iv and irinotecan $150 \mathrm{mg} / \mathrm{m}^{2}$ iv on day 1 , $6 \mathrm{~S}$-folinic acid $250 \mathrm{mg} / \mathrm{m}^{2}$ iv and fluorouracil $750 \mathrm{mg} / \mathrm{m}^{2}$ iv on day 2 , every 2 weeks. Response rate (RR) was assessed after a minimum of four cycles, and treatment continued up to 12 cycles.

Results Sixty-three patients were treated, with a median of eight (range 1-12) cycles/patient. Two complete and 19
\end{abstract}

SICOG trial 0405, EudraCT number 2006-0066869-16.

P. Comella $(\bowtie) \cdot$ R. Casaretti $\cdot$ C. Sandomenico $\cdot$ L. Franco

Unit of Medical Oncology,

Department of Gastrointestinal Tumours,

National Tumour Institute, Via Mariano Semmola,

80131 Naples, Italy

e-mail: pasqualecomella@libero.it

V. Lorusso

Unit of Medical Oncology, Oncology Institute, Bari, Italy

\section{Maiorino}

Unit of Medical Oncology, San Gennaro Hospital,

Naples, Italy

\section{Cannone}

Unit of Medical Oncology, City Hospital, Canosa, Italy

B. Massidda

Chair of Medical Oncology, University of Cagliari,

Cagliari, Italy

C. Putzu

Chair of Medical Oncology, University of Sassari,

Sassari, Italy partial responses were registered (RR 33\% [95\% CI, 2246\%]). Median progression-free survival was 7.5 (95\% CI, 5.6-9.4) months, and median overall survival was 12.1 (95\% CI, 10.8-13.4) months. Most common grade $\geq 3$ toxicities were neutropenia (59\%), febrile neutropenia (7\%), vomiting (20\%), and diarrhoea (10\%). All-grade neurotoxicity affected $33 \%$ of patients.

Conclusions Oxaliplatin, irinotecan, and fluorouracil/folinic acid administered every 2 weeks are safe and active in advanced gastric cancer.

Keywords Gastric cancer · Fluorouracil · Irinotecan · Oxaliplatin · Triplet regimen

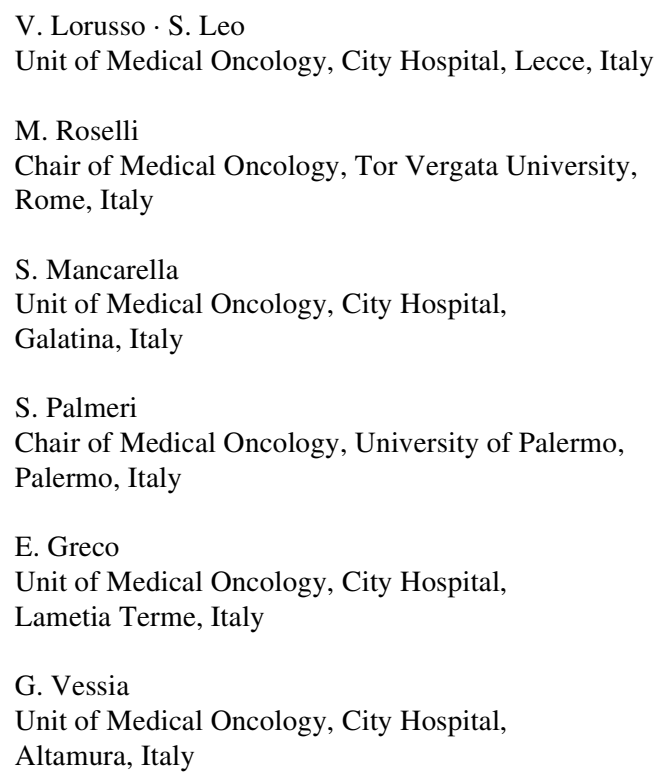




\section{Introduction}

Gastric cancer is still a significant global health problem [1]. In metastatic patients, chemotherapy may provide a substantial palliation, and can improve survival and quality of life compared to best supportive care [2], and fluorouracil, alone or associated with folinic acid, represents the cornerstone for the treatment of this disease [2]. A recent meta-analysis showed that combination regimens achieve better survival outcomes than fluorouracil monotherapy, and that regimens containing fluorouracil, anthracyclines and cisplatin are the most effective [3]. Indeed, epirubicin, cisplatin and infusional fluorouracil (ECF) regimen is one of the most widely used in Europe, while in US the standard regimen has remained for a long time cisplatin and infusional fluorouracil (CF) [2, 4-7].

Activity of irinotecan in advanced gastric cancer was seen in $18-23 \%$, regardless of prior chemotherapy $[8,9]$. Irinotecan has shown synergistic or additive activity with fluorouracil in vitro and in vivo [10-13]. Recently, weekly irinotecan plus infusional fluorouracil/folinic acid (IFF) was randomly compared with cisplatin plus fluorouracil given every 4 weeks. The IFF regimen produced a greater response rate (RR) (32 vs. $25 \%$ ), and demonstrated a marginally significant superior progression-free survival (PFS) (5.0 vs. 4.2 months, $P=0.088$ ), but not overall survival (OS) (9.0 vs. 8.7 months) in comparison with the reference regimen, showing a better toxicity profile [14].

The combination of oxaliplatin and fluorouracil/folinic acid has been extensively investigated in metastatic gastric cancer patients [15-20]. Recently, a trial comparing oxaliplatin, fluorouracil and folinic acid versus cisplatin, fluorouracil and folinic acid showed greater RR (34.8 vs. $24.5 \%)$, and a trend towards improved PFS (5.8 vs. 3.9 months, $P=0.077$ ) but not OS (10.7 vs. 8.8 months) for the oxaliplatin-arm, with less serious adverse events ( 9 vs. 19\%) [21]. Interestingly, a schedule-dependent interaction has been observed for oxaliplatin followed by fluorouracil, which was more synergic than the reverse sequence in cancer cell lines, either sensitive or resistant to fluorouracil [22]. In addition, in vitro experiments have demonstrated that the oxaliplatin and fluorouracil combination is more cytotoxic when fluorouracil is utilized as a short rather than prolonged exposure [23].

Synergistic cytotoxicity has been observed on human gastric cancer cell lines exposed to oxaliplatin followed by SN-38 (the active metabolite of irinotecan) [24]. Moreover, non-overlapping dose-limiting toxicities of oxaliplatin and irinotecan justify their combination in clinical practice. Indeed, some phase II trials on oxaliplatin and irinotecan have shown promising activity (RR 50-58\%, PFS 5.35.5 months) $[25,26]$.

This background represents a strong rationale for combining all these three active drugs in the treatment of advanced gastric cancer patients. We have already assessed this triplet in pre-treated patients with metastatic gastrointestinal carcinomas [27, 28]. Here we report on the safety and activity of this regimen in untreated gastric cancer patients.

\section{Patients and methods}

Patient selection and initial work-up

The aim of this study was to estimate the RR of oxaliplatin, irinotecan, and fluorouracil/folinic acid in advanced gastric cancer patients. Secondary end-points were safety, failurefree survival (FFS), PFS, and OS of patients.

Eligibility criteria were as follows: histologically proven diagnosis of adenocarcinoma of the stomach or gastrooesophageal junction; age $>18$ years; performance status (PS) $\leq 2$ of the Eastern Cooperative Oncology Group (ECOG) scale; unresectable or metastatic disease; measurable lesion(s); no previous exposure to palliative chemotherapy; discontinuation of fluorouracil-based (Machover or De Gramont regimen) adjuvant treatment for at least 6 months; absolute neutrophil count (ANC) $\geq 2,000 \mathrm{~mm}^{-3}$, platelet (PLT) count $\geq 100,000 \mathrm{~mm}^{-3}$, haemoglobin level $\geq 9.5 \mathrm{~g} / \mathrm{dl}$; bilirubin level $\leq 1.5 \times$ upper normal limit (UNL), serum alanine-aminotransferase and aspartate-aminotransferase $\leq 2.5 \times$ UNL in the absence of liver metastasis, or $\leq 5 \times$ UNL in the presence of liver metastasis; normal renal function. Exclusion criteria were: life expectancy $<12$ weeks; uncontrolled metabolic disorders or active infection; severe cardiac arrhythmia, uncontrolled congestive cardiac failure, severe ischemic heart disease, or acute myocardial infarction in the last 6 months; cerebral metastasis; other concomitant or previous malignant tumour. Patients gave written informed consent to participate into this study, which was approved by the Independent Ethics Committee of the National Tumour Institute of Naples.

At entry, physical examination, blood cell count with ANC and PLT counts, routine biochemistry, chest x-ray, and ECG were carried-out. Esophagogastric endoscopy was performed when indicated. Measurable lesions were assessed with CT or MRI scan.

\section{Treatment plan}

Oxaliplatin $85 \mathrm{mg} / \mathrm{m}^{2}$ iv (2-h infusion), followed by irinotecan $150 \mathrm{mg} / \mathrm{m}^{2}$ iv (60-min infusion) on day 1 , and $6 \mathrm{~S}$-folinic acid $250 \mathrm{mg} / \mathrm{m}^{2}$ iv (2-h infusion), followed by fluorouracil $750 \mathrm{mg} / \mathrm{m}^{2}$ iv (bolus) were given on day 2. Cycles were repeated biweekly for a minimum of four cycles, and up to 12 cycles. Prophylactic treatment with anti-HT3 for emesis, and with atropine for cholinergic syndrome, was mandatory. 
No prophylactic granulocyte colony-stimulating factor (G-CSF) was prescribed, but its use was mandatory for treating febrile neutropenia, and for preventing further episodes in subsequent cycles.

Treatment was recycled in the presence of ANC $\geq 1,500 \mathrm{~mm}^{-3}$ and PLT count $\geq 100,000 \mathrm{~mm}^{-3}$, and recovery of any extra-haematological toxicity. After an episode of grade 4 haematological toxicity, or grade $\geq 3$ non-haematological toxicity, subsequent cycles were administered with a $25 \%$ dose-reduction of all cytotoxic drugs. The same dose-reduction was applied from initial cycle for patients aged $>70$ years, or having previously suffered from severe toxicity during adjuvant chemotherapy; if treatment was tolerated, doses could be escalated in subsequent cycles. In the presence of transient neurotoxicity, no oxaliplatin dosereduction was applied. In the presence of persistent neuropathy, oxaliplatin was reduced to $75 \%$, while it was definitely discontinued for grade 4 neurotoxicity.

\section{Assessment of toxicity}

Blood cell count was performed weekly, while biochemistry, clinical and neurologic examinations were performed at every cycle. Acute toxicity was graded according to WHO toxicity criteria [29], while neurotoxicity was graded according to Lévi scale [30], and the worst toxicity suffered by each patient during the whole treatment was recorded.

\section{Assessment of activity}

Measurement of disease lesions with CT or MRI scans was repeated after every four cycles, and response was classified according to WHO criteria [27]. Responses were confirmed after a minimum of 4 weeks from initial documentation. RR was calculated on patients treated with at least one cycle (intent-to-treat [ITT]) population, and on those receiving at least four cycles (per-protocol population [PPP]). FFS was calculated from the date of registration to the date of discontinuation of treatment for progression, toxicity, refusal, or death, whichever occurred first. PFS was calculated from the date of registration to the date of tumour progression, or death. OS was calculated from the date of registration to the date of death for any cause, or last follow-up. After discontinuation of study treatment, patients were followed every 2 months to assess the disease status and survival. Second-line treatment was left to the attending physician's choice.

\section{Statistical considerations}

Sample size was defined according to minimax design of Simon [31], selecting a 0.05 alpha error, and a 0.20 beta error. Minimum RR $\left(p_{0}\right)$ was set at $30 \%$, while the alternative hypothesis $\left(p_{1}\right)$ was a $45 \%$ RR. Consequently, more than 25 responses should be achieved in a total of 65 patients.

Descriptive statistics were reported as proportions with their exact $95 \%$ confidence intervals (95\% CI). Time-toevent probabilities were estimated using the Kaplan-Meier method [32], computing the median and 95\% CI.

\section{Results}

\section{Enrolment}

From July 2005 to March 2008, 65 eligible patients were included in this study by 11 SICOG investigators. Main characteristics are listed in Table 1. Notably, 28 (43\%) patients were aged $\geq 65$ years, and $16(25 \%)$ patients were

Table 1 Main characteristics of enrolled patients

\begin{tabular}{|c|c|c|}
\hline Characteristics & No. & $\%$ \\
\hline Eligible patients & 65 & 100 \\
\hline Males & 45 & 69 \\
\hline Females & 20 & 31 \\
\hline Median age (years) & 61 & \\
\hline Range (years) & $26-81$ & \\
\hline \multicolumn{3}{|l|}{ Site of primary } \\
\hline Stomach & 54 & 83 \\
\hline Gastroesophageal junction & 11 & 17 \\
\hline Previous surgery & 27 & 42 \\
\hline Previous adjuvant chemotherapy & 7 & 11 \\
\hline Locally advanced & 5 & 6 \\
\hline Metastatic & 60 & 94 \\
\hline \multicolumn{3}{|l|}{ Number of disease sites } \\
\hline 1 & 7 & 11 \\
\hline 2 & 19 & 29 \\
\hline $3+$ & 39 & 60 \\
\hline \multicolumn{3}{|l|}{ Site of metastasis } \\
\hline Lymph nodes & 40 & 62 \\
\hline Liver & 39 & 60 \\
\hline Lung & 16 & 25 \\
\hline Peritoneal & 16 & 25 \\
\hline Other $^{\mathrm{a}}$ & 7 & 11 \\
\hline \multicolumn{3}{|l|}{ Performance status } \\
\hline 0 & 30 & 46 \\
\hline 1 & 34 & 52 \\
\hline 2 & 1 & 2 \\
\hline Previous weigh loss $>5 \%$ & 32 & 49 \\
\hline Baseline CEA > $5 \mathrm{ng} / \mathrm{mL}$ & 33 & 51 \\
\hline Baseline CA $19.9>37 \mathrm{U} / \mathrm{mL}$ & 30 & 46 \\
\hline Baseline alkaline phosphatase $>\mathrm{UNL}$ & 18 & 28 \\
\hline
\end{tabular}


aged $\geq 70$ years. All but four patients had metastatic disease, and most $(60 \%)$ had $\geq 3$ sites. Significant weight loss was reported for nearly half of patients. All but one patient had an excellent (ECOG 0, 46\%) or good (ECOG 1, 52\%) PS.

\section{Treatment delivery}

Two patients, due to worsening of clinical conditions, did never receive study treatment, leaving 63 patients assessable for safety and efficacy. Overall, 525 cycles were delivered, with a median of eight (range 1-12) cycles/patient. Early drop-out (before four cycles) occurred in ten patients, because of progression (six cases), severe toxicity (two cases), or withdrawn of consent (two cases). Therefore, 53 $(84 \%)$ patients received at least four cycles as per-protocol. Table 2 reports the treatment disposition. Of note, nearly half of patients were treated with $\geq 10$ cycles. Fifty-three (84\%) patients went off treatment for progression, four (6\%) for toxicity, and six (10\%) for refusal due to subjective treatment intolerance. Treatment ranged from 0.5 to 9.1 months (median 4.8 months).

Twenty-two (35\%) patients initiated the treatment with reduced doses, because of age (16 cases) or previous severe toxicity from adjuvant chemotherapy (6 cases), while 15 (24\%) patients had a dose-reduction over the first four cycles. Absolute (and relative) median dose-intensity was $28 \mathrm{mg} / \mathrm{m}^{2}$ per week $(66 \%)$ for oxaliplatin, $50 \mathrm{mg} / \mathrm{m}^{2}$ per week $(66 \%)$ for irinotecan, and $259 \mathrm{mg} / \mathrm{m}^{2}$ per week $(69 \%)$ for fluorouracil. Median (range) cumulative doses of oxaliplatin, irinotecan, and fluorouracil were $516(47-767) \mathrm{mg} /$ $\mathrm{m}^{2}, 888(83-1,412) \mathrm{mg} / \mathrm{m}^{2}$, and 4,571 (273-9,771) $\mathrm{mg} / \mathrm{m}^{2}$, respectively.

\section{Toxicity}

Six (9\%) patients died within 2 months from their enrolment, all because of progression. No treatment-related fatality was reported. Main side effects of treatment are listed in Table 3. Severe neutropenia affected $56 \%$ of

Table 2 Treatment disposition

\begin{tabular}{lll}
\hline Treatment & No. & $\%$ \\
\hline Total delivered cycles & 513 & 100 \\
Median cycles/patient & 8 & \\
Range & $1-12$ & \\
Patients treated with $\geq 2$ cycles & 57 & 90 \\
Patients treated with $\geq 4$ cycles & 53 & 84 \\
Patients treated with $\geq 6$ cycles & 46 & 73 \\
Patients treated with $\geq 8$ cycles & 43 & 68 \\
Patients treated with $\geq 10$ cycles & 27 & 43 \\
Patients treated with $\geq 12$ cycles & 22 & 35 \\
\hline
\end{tabular}

Table 3 Main side effects reported during treatment

\begin{tabular}{lccl}
\hline Toxicity & \multicolumn{3}{l}{ WHO grade } \\
\cline { 2 - 4 } & $1-2 \%$ & $3-4 \%$ & All grade (\%) \\
\hline Neutropenia & 15 & 56 & 71 \\
Febrile neutropenia & 5 & 7 & 12 \\
Thrombocytopenia & 32 & 0 & 32 \\
Anaemia & 49 & 5 & 54 \\
Nausea/vomiting & 46 & 20 & 66 \\
Diarrhoea & 39 & 10 & 49 \\
Stomatitis & 14 & 0 & 14 \\
Alopecia & 25 & 15 & 40 \\
Fatigue & 14 & 0 & 14 \\
Neurologic & 29 & 2 & 31 \\
Hepatic & 8 & 2 & 10 \\
Renal & 0 & 2 & 2 \\
\hline
\end{tabular}

patients (grade 4, 35\%). It more often occurred in aged $\geq 65$ years $(69 \%)$ than in younger $(54 \%)$ patients. Febrile neutropenia occurred in only $7 \%$ of patients. Five patients received G-CSF during the treatment. Severe anaemia and thrombocytopenia were negligible. Grade 3 diarrhoea, and grade 3 neuropathy occurred in 10 and $2 \%$ of patients, respectively (no grade 4 was recorded). Despite prophylactic anti-emetic treatment, $66 \%$ of patients complained of some gastric disturbance after chemotherapy, but these symptoms were severe in only $20 \%$ of them.

\section{Activity}

A complete response (CR) was registered in a 55-year-old male with inoperable lung metastases and baseline CEA serum level of $253 \mathrm{ng} / \mathrm{mL}$, in whom disappearance of lung nodules and normalization of CEA level occurred after 2 months of treatment. CR persisted for 3 months; thereafter disease recurred, and the patient eventually died after 19.4 months. Another CR was achieved in a 63-year-old female with unresected gastric primary, nodal and peritoneal spread, who showed disappearance of disease after 5 months of treatment; she recurred and eventually died 9 months after initial therapy. Nineteen patients achieved a partial response; therefore, RR was 33\% (95\% CI, 22-46\%) in ITT analysis (Table 4), and 40\% (95\% CI, 26-54\%) in PPP analysis. Responses were registered after a median of 10 (range 4-36) weeks, and had a median duration of 30 (range 4-114 weeks) weeks.

In addition, four patients showed minor tumour shrinkage. Noteworthy, among non-responding patients, 8 of 20 (40\%) with baseline abnormal CEA or CA 19.9 serum level showed a decrease $>50 \%$ of these values during therapy.

Overall, tumour control (response or disease stabilization) was achieved in 51 (81\%; 95\% CI, 69-90\%) patients. 
Table 4 Activity according to intent-to-treat analysis

\begin{tabular}{lrr}
\hline Responses & No. & $\%$ \\
\hline Complete response & 2 & 3 \\
Partial response & 19 & 30 \\
Stable disease & 30 & 47 \\
Progressive disease & 6 & 10 \\
Not assessed & 6 & 10 \\
Treated patients & 63 & 100 \\
\hline
\end{tabular}

RR was not associated with age, sex, or PS. Indeed, RR was $31 \%$ in subjects aged $\geq 65$ years, and $35 \%$ in younger ones; similarly, RR was $31 \%$ in males, and $35 \%$ in females; it was $27 \%$ for patients having a PS of 0 , and $39 \%$ among patients having a PS $\geq 1$. Other pre-treatment characteristics did not adversely affect achievement of response.

Ten of $24(42 \%)$ patients, in whom dose-intensity was above the median value for all three drugs, achieved a response, as opposed to 11 of $39(28 \%)$ patients treated with a with a lower dose-intensity $(P=0.204)$.

Three of four patients with initially unresectable disease underwent radical gastrectomy; moreover, 14 patients received second-line chemotherapy (docetaxel alone four cases; docetaxel plus cisplatin one case; capecitabine five cases; ECF two cases; oxaliplatin, fluorouracil and folinic acid two cases).

At the time of this report, after a median potential follow-up of 22 months, median FFS was 6.2 (95\% CI, 3.9$8.5)$ months. At that time, 48 (74\%) patients had shown a disease progression. The estimated median PFS survival was 7.5 (95\% CI, 5.6-9.4) months (Fig. 1). Forty-two (65\%) patients eventually died, and the estimated median OS was 12.1 (95\% CI, 10.4-13.8) months; 1- and 2-year OS probabilities $( \pm$ SE) were $51 \%( \pm 0.7 \%)$ and $20 \%$ $( \pm 0.6 \%)$, respectively (Fig. 2).

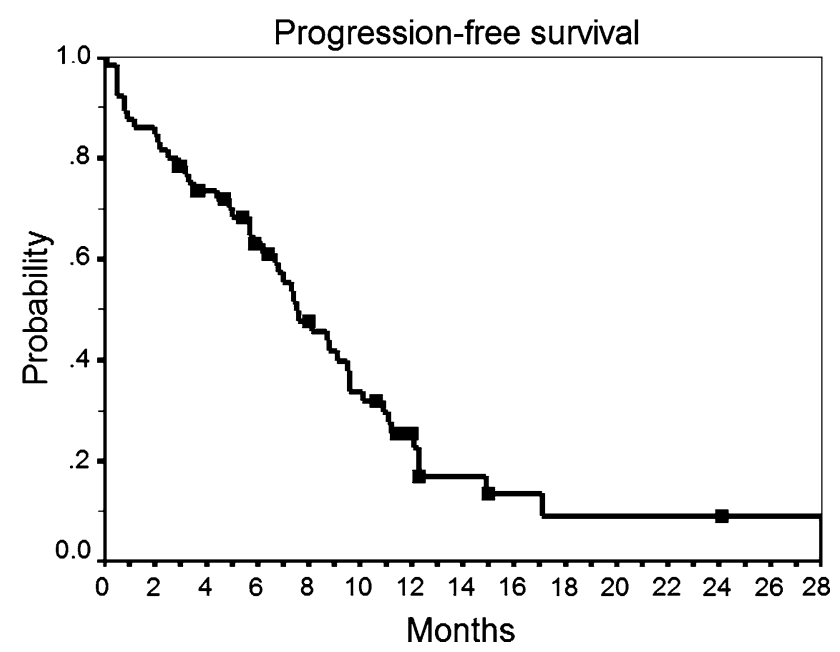

Fig. 1 Kaplan-Meier estimate of progression-free survival

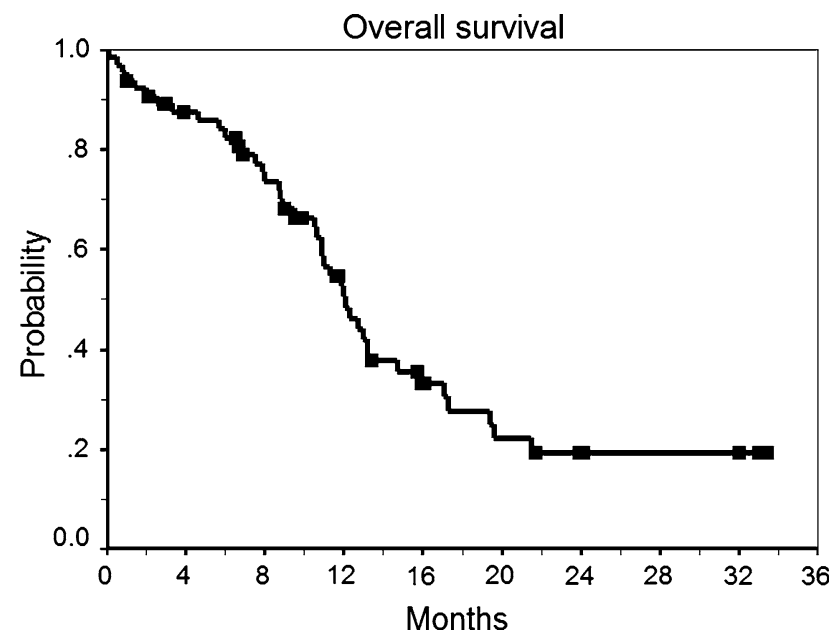

Fig. 2 Kaplan-Meier estimate of overall survival

\section{Discussion}

Treatment of advanced/metastatic gastric cancer remains still challenging. For many years, fluorouracil and cisplatin have been the only active agents for this disease, and a doublet of these compounds has been the reference regimen for treating these patients [1]. However, considering the results of recent trials [5-7], triplets have gained consensus as a standard treatment for advanced gastric cancer $[2,4]$.

The triplet tested in our trial showed a 33\% RR according to intent-to-treat analysis, while this figure rose to $40 \%$ as per-protocol analysis. Although this activity was inferior to those achieved by other doublets [10-13, 15-20, 25, 26], and lower than that hypothesized in the study design, we would stress that this result was obtained in a series reflecting the gastric cancer population commonly seen in the clinical practice. Moreover, activity of our regimen was comparable in elderly and younger patients, and no other baseline demographic or clinical characteristic appeared to significantly affect probability of response.

Activity of our regimen was also mirrored by a long median PFS of 7.6 months, likely due to the high fraction $(81 \%)$ of patients achieving disease control. Median OS (12.1 months) was also longer than expected for this kind of patients, and the probability of being alive at 2 years was $20 \%$. In this regard, we would underscore that, considering the prognostic factors of our series, two-thirds of patients should have a moderate $(43 \%)$ or poor risk $(23 \%)$ score, which in a retrospective multivariate analysis were associated with median OS of 4.1 and 7.4 months, respectively [33].

For an indirect comparison, we would recall the performance of ECF regimen, which demonstrated superiority over the FAM in terms of RR (45 vs. $21 \%$ ), FFS (median 7.4 vs. 3.4 months), OS (median 8.9 vs. 5.7 months), and 
quality of life [5]. Also with ECF or its variants, substituting oxaliplatin for cisplatin (EOF), or oral capecitabine for fluorouracil (ECX), median OS was inferior to 10 months. Only the double drug substitution in the EOX regimen was associated with a prolongation of median OS (11.2 months) in comparison with the ECF regimen, but the 2-year OS was still around $20 \%$. Moreover, no advantage in overall quality of life of patients was reported [6].

On the other hand, addition of docetaxel to cisplatin and fluorouracil (DCF regimen) demonstrated to significantly increase RR (37 vs. 25\%), PFS (median 5.6 vs. 3.7 months), and OS (median 9.2 vs. 8.6 months) of patients in comparison with the $\mathrm{CF}$ regimen. However, also in this case, the gain in median OS was marginal, with a more striking difference at 2-year observation (18 vs. 9\%), questioning the advantage of an early introduction in frontline of docetaxel, which surely increased the occurrence of febrile neutropenia and neutropenic infections, especially in patients aged $\geq 65$ years [7].

Tolerability of our triplet was acceptable, because only $16 \%$ of patients withdrew early from the study for toxicity or refusal, while $68 \%$ of patients received at least eight cycles. The most common severe side effect of this treatment was neutropenia (59\%); its occurrence compared favourably with that reported with DCF regimen (82\%) [7], but was greater than that produced by ECF (36-42\%) [5] or EOX (28\%) [6]. Notably, due to the low cumulative dosage of oxaliplatin actually delivered in our trial, grade 3 neurotoxicity was infrequent.

Due to preventive or mandated dose-reductions applied in our study to 35 and $24 \%$ of patients, respectively, doseintensity for all three cytotoxic drugs was suboptimal (around two-third of the planned one). Since there was a (non-significant) trend towards a higher RR for patients receiving a greater dose-intensity, we may speculate that tolerability, and possibly activity, of our regimen could be improved by the prophylactic delivery of G-CSF, at least in elderly patients.

Recently, Lee et al. assessed a three-drug combination of oxaliplatin, irinotecan, and 48-h infusion fluorouracil every 2 weeks in 48 metastatic gastric cancer patients with relatively favourable characteristics (median age 54 years; no patient aged $\geq 70$ years; $85 \%$ with only 1 or 2 involved organs). They reported a $68 \% \mathrm{RR}$, and a median PFS of 9.6 months [34]. Therefore, despite a higher RR, median PFS was only 2-month longer than that obtained in our study. Neutropenia (assessed every 2 weeks during treatment) affected $52 \%$ of patients, and incidence of grade 3 vomiting and diarrhoea was 44 and $10 \%$, respectively. Therefore, the all-infusional delivery of fluorouracil in this triplet did not seem safer than fluorouracil bolus. However, it remains to be explored whether oral capecitabine (mimicking a prolonged infusion of fluorouracil) could represent a preferable component of this triplet. Some preliminary experiences demonstrated the feasibility of such combination in metastatic colorectal cancer [35-37].

In conclusion, the triplet of oxaliplatin, irinotecan, and fluorouracil/folinic acid represents a tolerated and active treatment for advanced/metastatic gastric cancer patients, and it deserves comparison with other standard triplets. Moreover, it could be taken as a backbone on which to add molecularly target agents to be explored in this disease.

Conflict of interest statement No financial disclosures from any authors.

\section{References}

1. Wohrer SS, Radere M, Hejna M (2004) Palliative chemotherapy for advanced gastric cancer. Ann Oncol 15:1585-1595

2. Van Cutsem E, Van de Velde C, Roth A et al (2008) Expert opinion on management of gastric and gastro-oesophageal junction adenocarcinoma on behalf of the European Organisation for Research and Treatment of Cancer (EORTC) - Gastrointestinal Cancer Group. Eur J Cancer 44:182-194

3. Wagner AD, Grothe W, Haerting J et al (2006) Chemotherapy in advanced gastric cancer: a systematic review and meta-analysis based on aggregate data. J Clin Oncol 24:2903-2909

4. Cunningham C, Jost LM, Purkalne G, Oliveira J (2005) ESMO minimum clinical recommendations for diagnosis, treatment and follow-up of gastric cancer. Ann Oncol 16:22-23

5. Webb A, Cunningham D, Scarffe JH et al (1997) Randomized trial comparing epirubicin, cisplatin, and fluorouracil versus fluorouracil, doxorubicin, and methotrexate in advanced esophagogastric cancer. J Clin Oncol 15:261-267

6. Cunningham D, Starling N, Rao S et al (2008) Capecitabine and oxaliplatin for advanced esophagogastric cancer. N Engl J Med 358:36-46

7. Van Cutsem E, Moiseyenko VM, Tjulandin S et al (2006) Phase III study of docetaxel and cisplatin plus fluorouracil compared with cisplatin and fluorouracil as first-line therapy for advanced gastric cancer: a report of the V325 Study Group. J Clin Oncol 24:4991-4997

8. Köhne CH, Catane R, Klein B et al (2003) Irinotecan is active in chemonaive patients with metastatic gastric cancer phase II multicentric trial. Br J Cancer 89:997-1001

9. Farhat FS (2007) A general review of the role of irinotecan (CPT11) in the treatment of gastric cancer. Med Oncol 24:137-146

10. Moehler M, Haas U, Siebler J et al (2003) Weekly treatment with irinotecan, folinic acid and infusional 5-fluorouracil (IFL) in patients with advanced gastric cancer. Anticancer Drugs 14:645-650

11. Assersohn L, Brown G, Cunningham D et al (2004) Phase II study of irinotecan and 5-fluorouracil/leucovorin in patients with primary refractory or relapsed advanced oesophageal and gastric carcinoma. Ann Oncol 15:64-69

12. Bouché O, Raoul JL, Bonnetain F et al (2004) Randomized multicenter phase II trial of a biweekly regimen of fluorouracil and leucovorin (LV5FU2), LV5FU2 plus cisplatin, or LV5FU2 plus irinotecan in patients with previously untreated metastatic gastric cancer: a Federation Francophone de Cancerologie Digestive Group Study-FFCD 9803. J Clin Oncol 22:4319-4328

13. Kim ST, Kang WK, Kang JH et al (2005) Salvage chemotherapy with irinotecan, 5-fluorouracil and leucovorin for taxane- and cisplatin-refractory, metastatic gastric cancer. $\mathrm{Br} \mathrm{J}$ Cancer 23:1850-1854 
14. Dank M, Zaluski J, Barone C et al (2008) Randomized phase III study comparing irinotecan combined with 5-fluorouracil and folinic acid to Cisplatin combined with 5-fluorouracil in chemotherapy naïve patients with advanced adenocarcinoma of the stomach or esophagogastric junction. Ann Oncol 19:14501457

15. Louvet C, André T, Tigaud JM et al (2002) Phase II study of oxaliplatin, fluorouracil, and folinic acid in locally advanced or metastatic gastric cancer patients. J Clin Oncol 20:4543-4548

16. Al-Batran SE, Atmaca A, Hegewisch-Becker S et al (2004) Phase II trial of biweekly infusional fluorouracil, folinic acid, and oxaliplatin in patients with advanced gastric cancer. J Clin Oncol 22:658-663

17. Chao Y, Yeh KH, Chang CJ et al (2004) Phase II study of weekly oxaliplatin and 24-h infusion of high-dose 5-fluorouracil and folinic acid in the treatment of advanced gastric cancer. $\mathrm{Br} \mathrm{J}$ Cancer 91:453-458

18. De Vita F, Orditura M, Matano E et al (2005) A phase II study of biweekly oxaliplatin plus infusional 5-fluorouracil and folinic acid (FOLFOX-4) as first-line treatment of advanced gastric cancer patients. Br J Cancer 92:1644-1649

19. Lordick F, Lorenzen S, Stollfuss J et al (2005) Phase II study of weekly oxaliplatin plus infusional fluorouracil and folinic acid (FUFOX regimen) as first-line treatment in metastatic gastric cancer. Br J Cancer 93:190-194

20. Kim DY, Kim JH, Lee SH et al (2003) Phase II study of oxaliplatin, 5-fluorouracil and leucovorin in previously platinum-treated patients with advanced gastric cancer. Ann Oncol 14:383-387

21. Al-Batran S-E, Hartmann JT, Probst S et al (2008) Phase III trial in metastatic gastroesophageal adenocarcinoma with fluorouracil, leucovorin plus either oxaliplatin or cisplatin: a study of the Arbeitsgemeinschaft Internistische Onkologie. J Clin Oncol 26:14351442

22. Placencia C, Taron M, Abad A, Rosell R (2000) Synergism of oxaliplatin (OXA) with either 5-fluorouracil (5FU) or topoisomerase I inhibitor in sensitive and 5FU-resistant colorectal cancer cell lines is independent of DNA-mismatch repair and p53 status. Proc Am Soc Clin Oncol 19:204a (Abstr 793)

23. Fischel J-L, Etienne M-C, Formento P, Milano G (1998) Search for the optimal schedule for the oxaliplatin/5-fluorouracil association modulated or not by folinic acid: preclinical data. Clin Cancer Res 4:2529-2535

24. Tanaka R, Ariyama H, Qin B et al (2005) Synergistic interaction between oxaliplatin and $\mathrm{SN}-38$ in human gastric cancer cell lines in vitro. Oncol Rep 14:683-688

25. Souglakos J, Syrigos K, Potamianou A et al (2004) Combination of irinotecan (CPT-11) plus oxaliplatin (L-OHP) as first-line treatment in locally advanced or metastatic gastric cancer: a multicentre phase II trial. Ann Oncol 15:1204-1209

26. Wöll E, Kühr T, Eisterer W (2008) Biweekly oxaliplatin and irinotecan chemotherapy in advanced gastric cancer. a first-line multicenter phase II trial of the Arbeitsgemeinschaft Medikamentöse Tumortherapie (AGMT). Anticancer Res 28:2901-2905

27. Comella P, Casaretti R, De Rosa V et al (2002) Oxaliplatin plus irinotecan and leucovorin-modulated 5-fluorouracil triplet regimen every other week: a dose-finding study in patients with advanced gastrointestinal malignancies. Ann Oncol 13:1874-1881

28. Comella P, Massidda B, Palmeri S et al (2006) Biweekly oxaliplatin plus irinotecan and folinic acid-modulated 5-fluorouracil: a phase II study in pretreated patients with metastatic colorectal cancer. Anticancer Drugs 7:985-992

29. Miller AB, Hoogstraten B, Staquet M, Winkler A (1981) Reporting results of cancer treatment. Cancer 47:207-214

30. Lévi F, Misset JL, Brienza S et al (1992) A chronopharmacologic phase II clinical trial with 5-fluorouracil, folinic acid, and oxaliplatin using ambulatory multichannel programmable pump. High antitumor effectiveness against metastatic colorectal cancer. Cancer 69:893-900

31. Simon R (1989) Optimal two stage design for phase II clinical trials. Control Clin Trials 10:1-10

32. Kaplan ES, Meier P (1958) Non parametric estimation for incomplete observations. J Am Stat Assoc 53:557-580

33. Chau I, Norman AR, Cunningham D, Waters JS, Oates J, Ross PJ (2004) Multivariate prognostic factor analysis in locally advanced and metastatic esophago-gastric cancer-pooled analysis from three multicenter, randomized, controlled trials using individual patient data. J Clin Oncol 22:2395-2403

34. Lee J, Kang WK, Kwon JM et al (2007) Phase II trial of irinotecan plus oxaliplatin and 5-fluorouracil/leucovorin in patients with untreated metastatic gastric adenocarcinoma. Ann Oncol 18:88-92

35. Bajetta E, Celio L, Ferrario E et al (2007) Capecitabine plus oxaliplatin and irinotecan regimen every other week: a phase I/II study in first-line treatment of metastatic colorectal cancer. Ann Oncol 18:1810-1816

36. Fornaro L, Masi G, Bursi S (2008) A dose finding and pharmacokinetic study of capecitabine in combination with oxaliplatin and irinotecan in metastatic colorectal cancer. Cancer Chemother Pharmacol, Oct 7. (Epub ahead of print)

37. Maroun JA, Jonker D, Cripps C, Goel R, Lister D, Chiritescu J (2007) Encouraging results from a phase I study of capecitabine, oxaliplatin and irinotecan as first-line therapy in patients with metastatic colorectal cancer (MCRC). J Clin Oncol, 2006 ASCO Annual Meeting Proceedings, Part I, vol 25, No. 18S (June 20 Supplement), 4086 